\title{
A Unified Account of the Discourse Function of the Chinese Particle "ne"
}

\author{
Wu Guo *
}

\begin{abstract}
This paper presents a unified account of the discourse function of the Chinese particle ne based on synchronic and diachronic studies of the function of the particle in statements, in questions and as a theme marker under an interactional and cognitive framework for discourse markers. It proposes that the particle ne has a general function of hearer engagement for Common Ground (CG) negotiation. The use of ne in discourse serves as an instruction for the hearer to pay special attention to a discrepancy in the perceived CG shared by the speaker and hearer in a conversation that the speaker thinks is highly relevant to the current interaction and needs to be negotiated and resolved. And it is the interplay between this core function of $n e$ and the propositional content of the host utterance in a specific context that generates the various interpretations in the literature associated with the use of $n e$. This core function of the particle $n e$ is rooted in the two core properties of existence marking and discourse-continuation marking, which pertain to its two origins - the locality marker $l i$ and the discourse continuation marker $n i-i n$ Middle Chinese (201-1000 CE) and Early Mandarin (1001-1900).
\end{abstract}

Key words: the Chinese particle ne, existence, discourse-continuation, Common Ground negotiation

\section{Introduction}

The particle $n e$ in Chinese is among the most frequently discussed topics in the literature. The particle occurs in the utterance-final position in both statements and questions, as well as within an utterance, and has been analyzed as having different functions according to its position and the utterance types in which it occurs. This paper attempts to formulate a unified account of the discourse function of the particle on the basis of the findings of my recent research into its function in statements, questions and truncated questions (Wu 2005a, 2005b, 2006).

I agree with $\mathrm{Li}(1999)$, Chu $(2002,2006)$ and $\mathrm{Xu}(2008)$ on their treatment of the particle

\footnotetext{
* Wu Guo: ITRG, School of Humanities and Languages, University of Western Sydney, Locked Bag 1797, Penrith South DC 1797, Australia. E-mail: g.wu@uws.edu.au.
} 
$n e$ as a discourse marker. And it is my hypothesis that the general function of the discourse marker $n e$ is hearer engagement for common ground (Clark 1996) negotiation, i.e. by using $n e$, the speaker instructs the hearer to pay special attention to the existence of the situation indicated by the host utterance so that shared common ground can be adjusted with regard to the current interaction. The purpose of this paper is to examine how this hypothesis applies to the particle in its three seemingly different uses in statements, in questions, and within an utterance. The paper is organized as follows. After this brief introduction, Section 1 reviews previous studies on the particle and Section 2 describes the theoretical framework under which the present research was carried out. Sections 3, 4 and 5 discuss the particle's function in statements, in questions and within an utterance respectively. My version of the general function of the particle will be proposed in Section 6 and conclusions will be presented in Section 7.

\section{Previous studies}

Previous studies of the particle ne have reflected the debate between what are called 'meaning maximalists', who attribute a particle's variation in use to the semantics of the particle and specify a number of different senses to it, and 'meaning minimalists', who try to isolate a unitary core meaning from various uses of a particle (Mosegaard Hansen 1998: $85-86)$.

\subsection{The maximalist approach}

Among scholars taking a maximalist approach are (Ding 1961), Chao (1968), Lü (1980), Zhu (1982), Liu et al (1983), and Yu et al (1998). Chao (1968) distinguishes seven meanings of $n e$ (including $n a$ ): four in statements, i.e. continued state, assertion of an equaling degree, interest in additional information, and mild warning; two in questions, i.e. indicating questions in a context, and questions with a specific point; and indicating a deliberate pause. Lü (1980) has reduced the particle's meanings to four: question marker in non-general questions, indicating facts with emphasis, continued state, and pause within a sentence, as exemplified in (1):

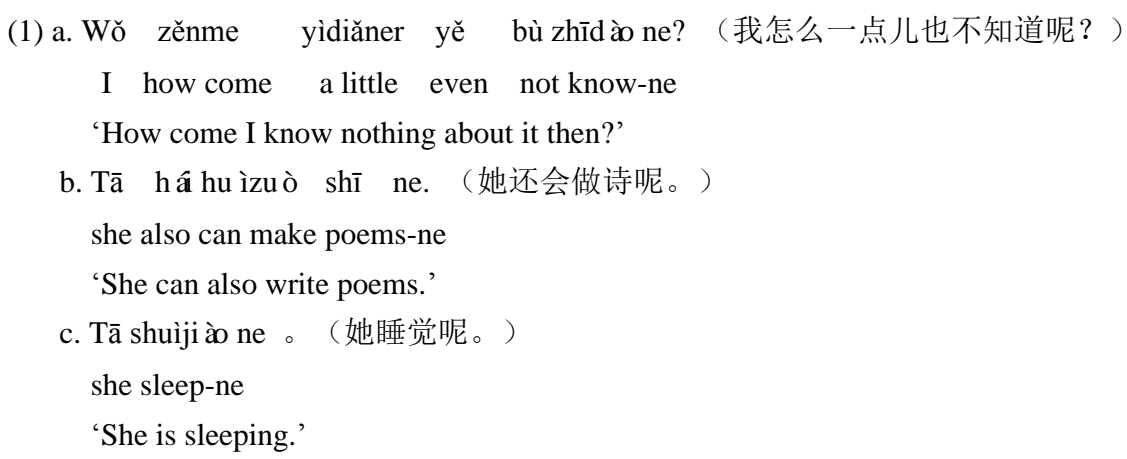


A Unified Account of the Discourse Function of the Chinese Particle "ne"

d. Rújīn ne, kě bù bǐ wăngcháng le. (如今呢, 可不比往常了.)

nowadays-ne, [emphasis] not as before-le.

'Nowadays, things are not the same as before.'

Zhu's (1982) and Yu et al's (1998) categorizations of the particle's meanings are in agreement with Lü's. Liu et al (1983) have dropped continued state, and listed three meanings for the particle: question marker, indicating affirmation in statements, and pause within a sentence. These maximalist views have been influential and are widely used in Chinese language teaching as well as academic discussions. Yet, as shown above, in this approach $n e$ in different contexts is given a different meaning/function, which is inevitably the result of the interaction between the function of ne and its context, not the meaning/function of ne alone. Tsao (2000) and Qi (2002) have criticized such analyses on the basis that they can hardly isolate the meaning of the particle from its context, and offer students little help in understanding and using the particle properly.

However, scholars adopting this approach have made insightful observations on the interactive nature of the particle and its involvement of the hearer. For instance, Lü (1982: 264) points out that $n e$ is used to make the listener believe that what is said is indeed factual, and has the effect of adding 'listen', 'believe me', or 'I'm telling you' to the speaker's statement. Similarly, Ding (1961:215) writes that "ne has the function of indicating facts and convincing the listener of this. It focuses on making the hearer believe, sometimes with an emphatic effect, " This, in my view, is an essential function of $n e$ and will be incorporated into my analysis.

\subsection{The minimalist approach}

On the other hand, many linguists have tried to uncover a unitary core meaning of the particle in various contexts. The different views in this camp of its function, discussed separately below, characterize the core meaning of $n e$ as something around reminding, as more interactive, as factual, or as diachronic and polysemic.

1.2.1 Reminding

$\mathrm{Hu}$ (1981: 417-418) insists on isolating the particle's meaning from its context and points out that neither continuation nor interrogative mood is part of the particle's meaning. Instead, he proposes that $n e$ invites the listener to pay special attention in what is said to a certain point, which is often marked by emphatic stress. Following $\mathrm{Hu}$, Shao (1989) proposes that the basic function of $n e$ is to remind the listener of something that is expressed by the word or phrase that carries the sentence stress and that usually precedes $n e$. In this, the particle signals "intense inquiry" in questions, and serves as a topic marker when used within a sentence. The function of $n e$ as proposed by $\mathrm{Hu}$ and Shao has made prominent its interactive nature and is able to account for most uses of ne. However, reminding is still one of the many contextual interpretations of its use, and is too specific to 
cover all its uses in discourse. For example, it cannot explain the use of $n e$ in the initial move $(2 \mathrm{a})^{(\mathbb{1}}$ in the following exchange in a TV series since the father doesn't need to be reminded of what he himself is doing.

(Situation: the son comes home in the evening and sees his father drinking alone, as he often does.)

(2) a. Son: Hē zhe ne? (喝着呢?)

drink-zhe-ne

'Having a drink ?'

b. Father: Ng, huílai le? (嗯, 回来了?)

yes, come back-le

'Yes, you are back?'

Furthermore, their claim that what listeners are being reminded of is expressed by the word or phrase on which the sentence stress falls is questionable, as Shi \& Zhang (1995: 73) argue.

It is this line that King and Xu also follow in their analyses. King (1986: 27) proposes that $n e$ is a pragmatic discourse particle and is used by the speaker "for highlighting or evaluating certain portions of background information in the discourse and bringing them to the attention of the hearer in the speaker/hearer world". By doing so, the speaker makes "a metalinguistic comment" and expresses "his attitude toward the content of the utterance". King nevertheless acknowledges that "it may not be possible to isolate an atomic meaning for the attitudinal aspect of $n e " . \mathrm{Xu}(2008: 159)$ generalizes the function of $n e$ as activating a certain piece of new information on the basis of the background information shared by the hearer and speaker, and bringing this information to the hearer's attention. In statements ne signals the difference between what the speaker says and the hearer's claims or expectations, whereas in questions it makes explicit the point in question for the hearer to provide an answer to.

King's and Xu's versions of the particle's function have taken into account the background information interactants have, the ongoing discourse, and the speaker's attitude toward the content of the utterance, which are essential elements in understanding the use of $n e$ in different contexts. I will include these elements in a framework that emphasizes the shared common ground (Clark 1996) of interactants underlying the interaction, and argue that the use of ne signals, and is conditioned by, common ground negotiations (see Section 2).

\footnotetext{
(1) It should be noted that the particle in (2a) is a statement $n e$, though it can be followed by a question marker. As a question, (2a) is in fact a general question with the question word ma deleted. Since ne questions are incompatible with $m a$ questions, the particle in (2a) can only be a statement $n e$. As a greeting, (2a) is uttered with a falling tone and it is actually inappropriate for the question word $m a$ to occur.
} 
A Unified Account of the Discourse Function of the Chinese Particle "ne"

\subsubsection{Speaker-hearer interaction}

Alleton (1981) and Li \& Thompson (1981) both focus on the speaker's intention in using ne. Alleton (1981: 111) proposes that the basic function of ne is to show "the speaker's appeal to his listener's or (listeners') active participation", which is clear in questions, but is to be interpreted differently in statements. Where a cognition verb is used, "the speaker suggests that the problem being discussed is not solved," and "ne takes the place of, or announces, a subsequent question; in other cases, $n e$ indicates that the speaker puts down this statement as a milestone in the course of the discussion." But in statements with a verb of quality as the predicate, "ne appears only as a simple indication of degree", and is thus excluded from this basic function. In contrast, Li \& Thompson (1981: 300) claim that ne indicates the speaker's response to the hearer's claim, opinion and expectation. Alleton's and $\mathrm{Li} \&$ Thompson's formulations have both captured the essence of $n e$ as a conversational particle. While Li \& Thompson's account explains the fact that $n e$ is overwhelmingly used in a reactive move, Alleton's observation that the use of $n e$ is "never disconnected from the previous discourse and from that following" (1981: 111) reveals an essential feature of the particle: indicating discourse continuation. It is along this line that Chu (2006) has developed his proposal for a unified account of the particle's discourse function, which will be discussed later.

However, Alleton's and Li \& Thompson's formulations only partially reflect the general function of the particle, with the former focusing on the effect on the hearer and the latter on the motivation of the speaker. Alleton has separated out one use of ne, which she claims is "a simple indication of degree," and in her analysis of other uses under her proposed function the use of ne with cognitive verbs does not have much in common with its use in what she terms 'other cases'. And Li \& Thompson do not take into account the cases where $n e$ is used in an initiative move, as in example (2) above, where no specific hearer's claims or expectations are involved, or what is involved are the expectations of a person other than the hearer.

\subsubsection{Fact}

Shi \& Zhang (1995) propose that the basic function of ne is to indicate facts or reality, which unifies the three functions in statements recognized by Lü (1980): indicating facts with emphasis, continued state, and pause within a sentence. These functions are, in fact, expressed by some elements in the utterances other than $n e$. They further argue that $n e$ in questions expresses exactly the same meaning of being factual as it does in statements. In Wh-questions ne asserts as a fact the part of the question to which the speaker thinks she knows the answer, and the question word marks the interrogation. Shi \& Zhang argue that is why they have not collected any questions formulated by a single Wh-word plus ne, and also why $n e$ is incompatible with the question marker $m a$ that marks a general question 
with no presupposed 'factual' portion to be asserted. They support their claim by pointing to the fact that ne is also incompatible with imperatives associated with unrealized situations as well as with exclamations indicating subjective feelings.

This function of $n e$ proposed by Shi \& Zhang captures one of its essential features: the association of $n e$ with an existing fact, which has been generally recognized by Chinese linguists (Chao 1968, Ding 1961, Lü 1980), and, in my view, should be included in any successful generalization of the particle's function. I fully agree with these authors' position for a unified interpretation of the particle, but disagree with them on three points.

Firstly, with regard to the notion of fact or reality, Shi \& Zhang (1995: 74) argue that the core use of $n e$ as indicating facts is incompatible with future time; hence (3a) is unacceptable.

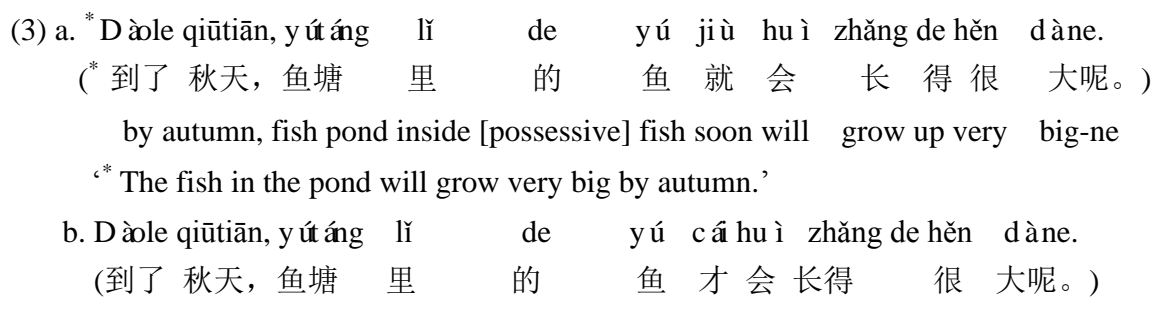

by autumn, fish pond inside [possessive] fish then will grow up very big-ne

'The fish in the pond won't grow very big until autumn.'

The problem with (3a), however, is the incompatibility of $n e$ with $j i u^{(\mathbb{1})}$ in the given context, not with using ne with future time. Thus (3b) becomes natural once jiu is replaced by cai, as shown above. Contrary to Shi \& Zhang's claim, information expressed by a ne utterance can be in future time. Intentions and plans for future events do go with ne because intentions and plans do exist in one's subjective world, even though future events do not. Examples in (4) are from everyday conversations.

(4) a. (Replying to an invitation to go out at the weekend)

Bù xíng, zhōumò wǒ hái xiě lùnwén ne.

(不行, 周末 我还写论文呢。)

no, weekend I yet write essay-ne

'No, I can't. I have to write my essay at the weekend.'

b. (Replying to a query about whether your child might want to be a teacher)

Rénjiā jiānglái yào dāng kēxuéjiā ne.

(人家 将来 要当科学家 呢。)

he future want be scientist-ne

'He wants to be a scientist.'

(1) Jiu goes with the particle le, whereas ne goes with cai. Cf. Ohta (1987). 
This shows that $n e$ not only indicates factual events that have happened, it also indicates schedules, plans and wishes that are perceived as existing realities. What is not compatible with $n e$ is not simply a future time, but whether a situation can be perceived as existing, hence $n$ 's incompatibility with the future time marker jiang that implies the present non-existence of a situation.

Secondly, I am not satisfied with Shi \& Zhang's claim that $n e$ in a Wh-question marks the factual part of that question known to the speaker. According to them, ne is not used when a question consists of a single Wh-word since there is nothing in the question known to the speaker. Obviously this cannot account for the famous line of the comedian Xiao Shenyang (Little Shenyang), "Weishenme $n e$ ?" (Why $n e$ ?), in his skit at the 2009 Chinese New Year's Eve gala celebrations, and other uses of "Wh-word + ne?" in daily conversations. In my view, since $n e$, as an utterance-final particle, encompasses the whole utterance, it is only reasonable to expect that it marks the whole question rather than a certain portion of it, and I will pursue this in my discussions later.

Finally, my overall criticism of this approach is to do with the lack of an interactive element in the function of a purely conversational particle. As is well known, ne does not appear in reports, scientific articles or expository writings (Alleton 1981, Li \& Thompson 1981), which is precisely the context where facts and reality are dealt with. The reason it does not occur there is because of the lack of the interaction that occurs in conversations.

\subsubsection{Diachronic and polysemic approach}

Tsao (2000) and Qi (2002) have examined the particle in a diachronic approach, tracing the grammaticalization of different meanings of $n e$ from its original core meaning. Tsao (2000) has identified five meanings of $n e$ in modern Chinese: unchanged state, topic marker, uncertainty/question, truncated question, and inconclusiveness. He claims that the meaning of an unchanged state is the core from which the other meanings have been derived directly or indirectly. On the other hand, Qi (2002) focuses on the modality of the particle and recognizes six types of marking: question, rhetorical question, puzzlement, exclamation, affirmation, and pause. According to him, $n e$ 's function as a question marker is the very source from which all other uses have been derived.

Tsao (2000) and Qi (2002) have greatly enriched the research on $n e$ by trying to reveal the underpinnings of the synchronic polysemy of ne through its semantic change over time. Their specific descriptions of the process, however, do not all seem to be supported by historical data. As I have mentioned elsewhere (Wu 2005b), ne in so-called truncated questions, which Tsao says is derived from its use as topic marker, was among the earliest

\footnotetext{
(1) Little Shenyang is a popular skit actor who said "Weishenme NE?" (Why NE?) many times in his debut at the 2009 Chinese New Year's Eve gala celebrations.
} 
uses of the particle before its topic-marking function developed. And according to Cao (1995), Qi (2002), and Jiang (2005), the origin of the affirmative $n e-l i-$ and that of the interrogative $n e-n i-$ actually first appeared at about the same time in the Tang and the Five Dynasties (seventh to tenth centuries) and, according to Jiang (1986), had been represented by the same word er even earlier in the Han Dynasty (second century BCE to second century CE).

The most recent and comprehensive account of the particle's common core is provided in Chu (2006). By means of its close counterparts in English, Chu has discovered two core properties: the necessity to look back for contrast and the demand for continuation. He lists three primary functions - interrogation, reminding, and topic marking - and two secondary functions - (marking) truncated question and intense inquiry - that are derivable from these two properties. He excludes various other meanings attributed to $n e$ in the literature such as idea development, puzzlement, unchanged state, uncertainty and inconclusiveness since they are interpretations of a ne utterance through propositional content and context. Chu's discourse-oriented approach has enabled him to capture the core of the particle's function in terms of discourse forces rather than anything lexical or syntactic. I endorse Chu's analysis and the way he distinguishes between the common core of the particle, functions derivable from it and interpretations through propositional content and context.

However, I would like to point out that Chu's analysis focuses on the particle's contribution to the textual aspect of discourse without giving enough attention to its contribution to the interpersonal aspect. Discourse continuation is indeed a core property of $n e$, but it is also a core property of many other discourse markers. To specify how ne is different from other markers, there needs to be a semantic element, from which various shades of the speaker's attitude, as described in the literature, are derivable. Chu correctly points out that meanings associated with ne utterances such as puzzlement are derived through propositional content and context, but I would like to ask what exactly ne contributes to these meanings beyond marking discourse continuation. To this question I will try to provide an answer in my later discussion.

\subsection{Summary}

As demonstrated above, no single formulation has offered a satisfactory account for the function of the particle ne. While scholars adopting the contextual approach do not offer a common core in their interpretations, the unified accounts in the literature are either too narrow (Hu 1981, Shao 1989), too general (King 1986), incomplete (Alleton 1981, Li \& Thompson 1983), or lacking in the interactive element (Shi \& Zhang 1995). Furthermore, the use of the particle in the initiating move such as greetings is missing in the literature; hence it is not accounted for.

Collectively, however, previous studies have depicted a comprehensive repertoire of its 
function and interpretations in context for a promising generalization of the particle's common core function. As I see it, three concepts proposed in the literature are essential to such a generalization: asserting facts (Lü 1980, 1982, Shi \& Zhang 1995) at the semantic and cognitive level, the speaker's attitude and implications to the hearer associated with this assertion at the pragmatic and interactive level (Lü 1982, Ding 1961, Hu 1981, Shao 1989, King 1986, Alleton 1981, Li \& Thompson 1981), and continuation at the discourse level (Alleton 1981, Xu 2008, Chu 1999, 2006). I will incorporate these three concepts in my analysis and argue that the general function of the particle ne is hearer engagement, which, I hope, will be able to account for the particle's various uses in conversation discussed so far.

\section{Theoretical framework}

Discourse markers, or particles, have been studied in various theoretical frameworks macro-syntax, integrated pragmatics, discourse-structural framework, interactional sociolinguistics (Schiffrin 1987, 1994) and relevance theory (Sperber \& Wilson 1986, cf. Mosegaard Hansen 1998) — and from different perspectives - sentence-type, modality, speech act and discourse (cf. Li 1999) since the late 1960s. More recently, Mosegaard Hansen's (1998: 73-79) work on French discourse markers has taken a dynamic global approach, integrating a number of dynamic notions such as instructional semantics, polysemy, grammaticalization, conversation analysis, the emergent nature of discourse structure, and the construction and continual updating of mental representations in the pursuit of coherence. Berg \& Wu (2006) investigate the Chinese discourse $L E$ from an interactional framework in which verbal interactions are considered as joint activities in Clark's (1996) terms. In the attempt to generalize the particle's core function, this paper will incorporate into the framework of Berg \& Wu (2006) some essential elements in Mosegaard Hansen's (1998) global dynamic approach, such as instructional semantics, relevance theory and coherence in terms of mental representations.

As Berg \& Wu (2006) point out, Clark's (1996) view of verbal interaction as joint activity is essential to understanding the function of discourse markers, and a key concept related to this view is common ground (CG). $\mathrm{CG}$ is built up over time and has two aspects: communal $C G$ and personal $C G$. The first relates to the cultural community a person belongs to, the second to one's personal experiences with other people in that community (Clark 1996: 120). Since "everything we do is rooted in information we have about our surroundings, activities, emotions, plans, interests" (Clark 1996: 92; cf. Johnson-Laird 1983), verbal interactions as joint activities are rooted in CG. CG is a shared basis for communication and can be reflected upon (Clark 1996: 94-95). The essential feature of CG is that it is shared or it is believed to be shared. What is reflected upon can be shared but 
need not be; it can be personal or subjective, in which case it is not shared. Only when, after reflection, communication on the basis of CG proceeds can a personal reflection be made about CG (Clark 1996: 96).

In this view verbal interaction is a process whereby shared CG is enhanced. As conversation analysis reveals, meanings are being continually negotiated between interlocutors on a turn-by-turn basis, such that a turn in conversation will normally display the speaker's understanding of the previous turn by her interlocutor, this understanding being subject to revision by the hearer in the following turn (cf. Heritage 1984: 255-257). Whatever is confirmed in the revision will be built into the CG, and then the initial CG that formed the basis for the interaction will be enhanced to a new level. In this process discourse markers serve as a cue to coordinate the joint activity, and "fulfill an important function as linguistic 'road signs' guiding the hearer towards the interpretation of the discourse that the speaker intends" (Mosegaard Hansen 1998: 199).

In addition to CG, instructional semantics (Johnson-Laird 1977, 1983; Harder 1991, 1996), relevance theory (Sperber \& Wilson 1986) and coherence in terms of mental representations (Mosegaard Hansen 1998) are important notions for this study. An instructional view of linguistic semantics sees the meaning of a linguistic item as instructional or "procedural" in artificial intelligence terms rather than as representational. For instance, an instructional view would see the use of the Chinese particle le as an instruction to the hearer to update the shared CG with a new situation and act accordingly rather than activating the concept of a situation change (cf. Berg \& Wu 2006). Instructional semantics assigns a more prominent role to discourse markers, which are to guide the hearer in the search for optimal relevance in the process of verbal interaction.

Relevance theory (Sperber \& Wilson 1986) has boiled Grice's $(1971,1975)$ cooperative principle with four attendant maxims down to one overarching principle in terms of contextual effect. There are three kinds of contextual effect: deduction of contextual implications, the strengthening, and the weakening or deletion of an already existing assumption. The degree of relevance is determined on a cost/benefit basis: the greater the contextual effect an utterance has in a given context, the greater its degree of relevance; the smaller the cognitive effort needed to work out an interpretation of an utterance, the greater its degree of relevance. The degree of relevance is determined on a cost/benefit basis. Relevance is closely linked to the function of discourse markers (cf. Chu 2002) and underpins the overall coherence of discourse to be discussed below.

Strictly speaking, coherence is not a property of texts, but rather of mental representations, formed on the basis of text, context, and inferences from both during discourse comprehension (Mosegaard Hansen 1998: 176). A mental model (Johnson-Laird 1989: 249) of a discourse typically incorporates not only knowledge directly expressed in 
the discourse, but also background knowledge and inferences necessary to comprehension. As the discourse proceeds, the model is continually updated, and possibly revised, in the light of incoming information. Discourse markers in this process "function as instructions on how to process the content of their host utterance with a view to integrating it into a mental representation of the discourse. Markers will then often be used to signal that the speaker intends the host utterance to cohere in a specific way with the preceding discourse" (Mosegaard Hansen 1998: 196).

The views of verbal interaction as joint activities and of discourse comprehension as mental model construction complement each other, with the former approaching discourse from a global context of social interaction, and the latter focusing more specifically on how discourse is processed in the human mind. CG enhancement in verbal interactions, therefore, can be seen as resulting from the continual updating of the mental model of discourse. In this framework, discourse markers serve as a coordination device for CG enhancement that give instructions to the hearer on how the situation designated by the host utterance is relevant to the mental model of the discourse so far constructed.

The rest of this paper will examine what exact instructions the particle ne signals to the hearer in different contexts, and identify the common core that underpins these instructions.

\section{The discourse function of $n e$ in statements}

$\mathrm{Wu}$ (2005: 79) argues that in statements:

... the particle $n e$ has a general function of hearer engagement for CG negotiation. The use of $n e$ in discourse serves as an instruction for the hearer to pay special attention to the existence of the situation designated by the host utterance, which the speaker perceives as (missing in the context, but) highly relevant to the current interaction, typically contradicting the hearer's or supporting the speaker's claim or assumptions. And it is the interplay between this function of $n e$ and the content of the host utterance in a specific context that generates the various meanings associated with the use of $n e$ in the literature.

In this formulation, three notions underlie the common core of the particle's function: hearer engagement, CG negotiation, and existence of a factual situation. "Engage" here is used in at least three senses - "to occupy the attention of a person: He engaged her in conversation", "to attract and hold fast: The novel engaged her attention and interest", and "to enter into conflict with: Our army engaged the enemy" (dictionary.com) - to cover a wider range of the particle's uses than the traditional "reminding" (Shao 1989): from initiating a conversation, to drawing the hearer's attention to a factual situation, including "reminding", to contradicting the hearer's claim or expectation. And the purpose of the engagement is CG negotiation, which presupposes that the speaker perceives a difference 
in the shared CG being constructed between him and the hearer and is prepared to voice it and to make clear his attitude toward it.

The last of the three notions - existence - is a core concept of the particle's function. It is rooted in the lexical origin of $n e$ and its function as a discourse marker. Historically, the modern particle $n e$ in statements developed from the locality marker $l i$ (里), meaning "inside", which began to be used in the Tang dynasty (seventh to tenth centuries) at the end of an utterance to indicate the existence of a factual situation in a certain location (Ohta 1987: 350). Gradually, it began to occur in utterances where no locality of a factual situation was involved in the objective world (only an existence in the subjective world), to alert the hearer or catch the hearer's attention in a function Ohta (1987: 352) calls 精警 jingjing (warning), e.g.:

(5) Yǒu wǒ ne.

(有我呢。)

There is I ne. (to support or help...)

I'm here (if you need help), you know.

What I call hearer engagement is a natural stage of this historical development:

Inside $\rightarrow$ asserting locality $\rightarrow$ asserting existence $\rightarrow$ alerting $\rightarrow$ engaging hearer

Along this path of grammaticalization, the meaning of $l i$ (ne) has become increasingly general and subjective, and more discourse oriented, showing a unidirectional increase of subjectification, as proposed by Traugott (1995: 45-47). And the meaning of ne has "become increasingly based in the speaker's subjective belief state/attitude toward the proposition" (Traugott 1989: 35). It is ne's link with the notion of existence, objective or subjective, and the historical process of subjectification that determines that $n e$ is used to indicate or assert (objective or subjective) facts (Lü 1980, 1982, Shi \& Zhang 1995), and to signal a speaker's subjective belief state about the facts asserted. Strictly speaking, when tagged by $n e$, a statement is not a pure statement any more. It becomes "a metalinguistic comment" and expresses the speaker's "attitude toward the content of the utterance", as King has observed (1986: 27).

It is the notion of existence or, more accurately, its absence that motivates the use of $n e$ in verbal interactions, and thus determines the particle's pragmatic function. As an existence marker, ne is needed only when a situation to be marked is perceived by the speaker as missing in the context, but relevant to the current interaction. According to Sperber \& Wilson's (1986) relevance theory, in terms of contextual effect a hierarchy of situations where information is missing but is relevant in verbal interaction should be:

(a) Where there is an assertion, expectation, or situation in context that contradicts the information perceived by the speaker as relevant.

(b) Where the information perceived by the speaker as relevant is somehow 
unexpected or not being considered.

(c) Where the information is unavailable to the hearer and is to be provided by the speaker.

(d) Where the information is available to the hearer, but is not in the hearer's attention.

These match the findings in $\mathrm{Wu}$ (2005) where 10 out of the total 31 cases of ne utterances function as direct contradictions either to claims/expectations that have arisen in the context of the conversation, or to reality the speaker has just recognized, whereas 19 occur in the second move of the claim-support sequence to back up the speaker's position, most of which reveal factual situations that counter the hearer's expectations or are unexpected by the hearer. These 29 cases fall in situations (a) and (b) with strong assertive force, and the remaining 2 cases fall in (c). The low occurrence of the particle in (c) is to be expected, as this is a relatively neutral context where ne-marking is not as highly motivated as in contradicting or counter expectation, and its assertive force is less strong. At the bottom end of the scale in (d) are ne utterances initiating a conversation, which are not found in the limited data, but are frequently heard in everyday conversations, e.g. ${ }^{(1)}$

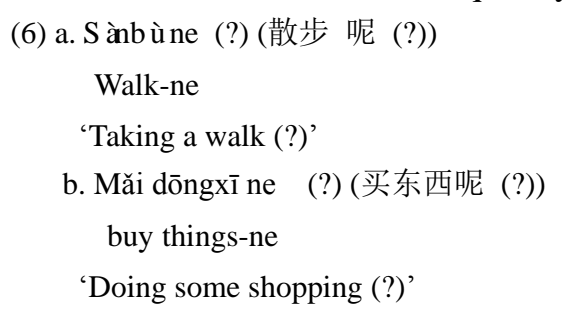

In such cases the function of the particle is to draw the hearer's attention to the speaker's communicative intent to engage her in conversation by addressing the obvious shared situational CG. It in fact serves as a kind of greeting. Obviously, the assertive force of the $n e$ utterance is weakest in this use.

To sum up, the core property of the particle $n e$ is hearer engagement, derived from its original function of existence marking. By tagging $n e$ to a statement, the speaker asserts the situation indicated by the utterance as factual, but missing in the context, and urges the hearer to adjust the shared CG accordingly by taking the situation into account. It is the interplay between $n e$ 's existence marking and the host-utterance content in context that determines the ranges of assertive force and speaker's attitude attributed in the literature to ne.

As shown above, the higher the contextual effect of a ne utterance, the higher the

\footnotetext{
(1) It should be noted that the particles in (6) are statement $n e$, though they can be followed by question markers. As questions, (6) are in fact general questions with the question word ma deleted. Since ne questions are incompatible with $m a$ questions, the particles in (6) can only be statement $n e$. As greetings, (6) are uttered with a falling tone and it is actually inappropriate for the question word ma to occur.
} 
relevance of the need to assert the existence of a certain factual situation, and the stronger the assertive force. The assertive force displayed by ne utterances reduces down along the hierarchy from contradicting, countering expectation, contrasting, to convincing, informing, and greeting. Meanwhile, the speaker's attitude associated with these speech acts also softens. However, whatever the attitude in a specific context, it is always derivable from the speaker's belief state about a factual situation missing in context. As a result, as Lü (1982: 264) points out, the use of the particle has, in general, the effect of adding 'listen', 'believe me', or 'I'm telling you' to the host utterance. It is clear that the primary difference between a ne utterance and its counterpart without $n e$ is the speaker's engagement of the hearer to negotiate a shared CG on the basis of which the current interaction takes place. This explains why $n e$ is a purely conversational particle.

\section{The discourse function of $n e$ in questions}

$\mathrm{Wu}$ (2005b: 74) proposes that the discourse function of $n e$ in questions is "marking discourse continuation" In general, the particle marks three kinds of relevance in questions: to the preceding discourse, to the CG of the interactants including the immediate situation, and to the speaker's train of thought, i.e. epistemic state. For instance, ne signals that the question is related to the preceding discourse in (7a), to the immediate situation in (7b), and to the speaker's train of thought in (7c):

(7) a. Fán: zhème shuō, tā zài zhè jǐ tiān jiù zǒu, jiūjìng dào shénme dìfāng qù ne?

(繁: 这么 说, 他在 这几天 就 走, 究竟 到 什么 地方去呢? )

Fan: so speak, he in the few days soon leave exactly arrive what place go-ne

'Fan: So, he is leaving any day soon. Where is he going to go then?'

b. Chōng: bàba, zěnme Lǔ Dàhăi hái zài zhèér děngzhe yào jiàn nín ne?

(冲: 爸爸, 怎么 鲁大海 还 在 这儿 等着 要 见您呢?)

Chong: father, how come Lu Dahai still in here wait want see you-ne

'Chong: Father, How come Lu Dahai is still here waiting to see you?'

c. Lǔ: yí, qíguài...wèishénme chuānghù hái guānshàng ne?

(鲁: 咦, 奇怪......为什么窗户 还关上呢?)

Lu: well, weird... why window still closed-ne

'Lu: Well, that's weird ... I wonder why the windows are still closed.'

A ne question, however, may well involve more than one, or all three, of these with different degrees for each, and the division is only a matter of degree. The question's link to the speaker's epistemic state is the least discussed of the three but is the most important for understanding the function of the particle in questions. If unable to find the link signalled by ne with the preceding discourse or situation, the hearer will assume the relevance is to the speaker's train of thought, which is also part of the interactional 
situation, but not shared. Such questions are often in the form of self-talk or soliloquy, indicating that the speaker is pondering the situation.

The three levels of relevance signalled by ne synchronically to the interactional context have, to a certain extent, reflected the particle's diachronic path of grammaticalization. Historically, the particle has developed from its origin er (Ohta 1987: 336), with the meaning of "the way of (something)" or "such", which was used in questions with a link to preceding discourse as well as in Wh-questions (Sun 1997: 35-36). However, all the earliest cases of er questions, including Wh-questions, found in Yang (1929), Jiang (1986) and Cao (1995) are elliptical, showing a link with preceding discourse. Therefore, discourse continuation as the core property of the particle is rooted in its original objective meaning indicating "the way of (something)". As it underwent grammaticalization, the particle's meaning became more and more subjective, discourse and epistemically oriented, moving from its original objective meaning "the way of (something)" or "such" to the preceding discourse, which is less objective, to the more subjective CG and to the speaker's train of thought or epistemic state, which is subjective. This process has exemplified what Traugott (1995: 32) calls "subjectification in grammaticalization" "whereby forms and constructions that at first express primarily concrete, lexical, and objective meanings come through repeated use in local syntactic contexts to serve increasingly abstract, pragmatic, interpersonal, and speaker-based functions".

If the hypothesis that there is a unidirectional increase in subjectification over time in semantic change (Traugott 1995) is in general correct, the particle ne should occur more frequently in questions reflecting higher degrees of subjectivity, and its primary function should be to signal a question's relevance to the speaker's epistemic state. And this is supported by the data.

A comparison of the ne questions in the play Leiyu (Thunderstorm), Act 2, and those in the novel Luotuo Xiangzi (Rickshaw Boy), sections 7-12, shows that only 12\% of all the Wh-questions in Leiyu are marked by ne, whereas in Luotuo Xiangzi there are almost four times as many (44\%). The high frequency of ne questions in Luotuo Xiangzi seems to be related to frequent descriptions of Xiangzi's state of mind, where the questions are not directed to a hearer, but to the speaker himself. As such self-directed questions are closely related to the speaker's train of thought, $n e$ is frequently needed to signal that relevance. In Leiyu, which is a play, characters frequently interact with little space for self-reflection, and therefore there are few self-directed questions. While in Luotuo Xiangzi 31 of 81 (38\%) Wh-questions are self-directed, in Leiyu only 2 of $134(1 \%)$ are. These statistics confirm the correlation between ne tagging and self-directed questions. This correlation has been further consolidated and specified by my further investigations into the distribution of ne questions in self-directed and hearer-directed Wh-questions, and the distribution of 
self-directed and hearer-directed questions in Wh-questions with and without ne tagging, as shown in the following tables:

Table 1. Distribution of Wh-questions in Luotuo Xiangzi, sections 7-12

\begin{tabular}{||l||c|c|c|c|c||}
\hline & Total & Self-directed & Ratio & Hearer-directed & Ratio \\
\hline \hline Wh-Q + ne? & $36(100 \%)$ & 30 & $83 \%$ & 6 & $17 \%$ \\
\hline Wh-Q? & $45(100 \%)$ & 1 & $2 \%$ & 44 & $98 \%$ \\
\hline \hline
\end{tabular}

Table 2. Distribution of self- and hearer-directed Wh-questions in Luotuo Xiangzi, sections 7-12)

\begin{tabular}{|l||c|c|c|c|c||}
\hline & Number & Wh-Q + ne? & Ratio & Wh-Q? & Ratio \\
\hline \hline Self-directed & $31(100 \%)$ & 30 & $97 \%$ & 1 & $3 \%$ \\
\hline Hearer-directed & $50(100 \%)$ & 6 & $12 \%$ & 44 & $88 \%$ \\
\hline Total & $81(100 \%)$ & 36 & & 45 & \\
\hline
\end{tabular}

These statistics indicate that most self-directed Wh-questions in this work are marked by the particle $n e$. Of all the Wh-questions with $n e, 83 \%$ are self-directed; of all self-directed Wh-questions, 97\% have ne. On the other hand, most hearer-directed Wh-questions are not marked by the particle: of all the Wh-questions without ne, $98 \%$ are hearer-directed; of all the hearer-directed Wh-questions, $88 \%$ do not have $n e$. There is thus no doubt that self-directed questions do attract the use of $n e$. And underlying this correlation is the particle's discourse-continuation function - to signal the relevance of the question to the speaker's train of thought. The various meanings attributed in the literature to the particle, such as "puzzlement" or different tones associated with ne questions, are all related to the link with the speaker's epistemic state signalled by the particle. These, in my view, are not the meaning proper of $n e$, but conversational implicatures derivable from the particle's discourse-continuation function in different contexts.

For instance, a speaker's puzzlement is derivable from the host question's relevance, signalled by $n e$, to the speaker's train of thought, which often implies that the speaker has been pondering the issue and is trying to find an answer now. However, it should be noted that puzzlement is not the basic meaning of $n e$ and does not necessarily apply to all $n e$ questions. For instance, in rhetorical questions, the relevance to the speaker's train of thought serves more to appeal to the hearer to ponder the question along the speaker's line of thinking than to express puzzlement.

As for the tone associated with ne questions, they have been described as being "intense enquiry" (Shao 1989: 174), "enhancing the interrogative force" (Kang 1998: 97), "mildly intense enquiry" (Ye 1994: 449), and "softening the tone of the question" (Shi \& Zhang 1995, Chen 1995). I agree with Shi \& Zhang on the softening effect of $n e$ in questions, but for different reasons. In my view, it is the particle's linking of the host question to its 
interactional context and the speaker's epistemic state that accounts for the softening effect. In general, the interrogative force of a question is reduced when the hearer's attention is drawn not only to the question itself, but also to the context in which the question has arisen. The situation is similar to taking a photo. An object is the focus of attention in a close-up but not when the camera zooms out to take in the background as well. Similarly, without $n e$, the focus of a question is the question itself; however, once $n e$ is used to signify the question's link with the context, there is less focus on the question itself, with a resultant reduction in interrogative force and an increased softening effect. On the other hand, the interrogative force of a ne question is further softened by the particle linking it to the speaker's train of thought, and its overwhelming use in self-directed questions, since these can be interpreted as somehow self-directed having a similar effect to an English question prefaced by "I wonder ....", makes the question less direct, thus reducing the pressure on the hearer to answer the question.

Finally, since the particle signals discourse continuation, it is expected that when such continuation is absent, or relevance is not considered, ne should not be used. An examination of Wh-questions without ne in Leiyu reveals that they occur under three circumstances: when questions are about new discourse participants or events, when the focus is on the question itself, and when the speaker expresses a firm belief or demand (cf. $\mathrm{Wu} 2005 \mathrm{~b}$ ). The first circumstance in general lacks discourse continuation and it is only reasonable to expect the absence of $n e$. And the second involves interrogations where information is the only focus of the question, such as police interrogations or questions addressed to someone at a registry. The third is often associated with rhetorical questions and requests where the speaker expresses a belief or demand firmly, leaving no room for discussion. The speaker doesn't need to engage the hearer or appeal to the hearer to follow his line of thinking. The use of the particle in such cases would soften the force of rhetorical questions and demands couched in the form of a question.

In all these cases without $n e$, discourse continuation is either absent or not considered due to the need to focus on the question itself. This demonstrates, from a different perspective, that discourse continuation is the basic function of the particle ne in questions.

\section{The discourse function of $n e$ as a theme marker}

In this section I follow Halliday (1985) and Zhang \& Fang (1996) in using the term "theme" for Halliday's ideational, interpersonal and textual constituents in the pre-verbal position rather than "topic", as widely used in the literature, which in general excludes interpersonal and textual elements.

The use of the particle ne as a theme marker is historically derived from its origin $n i$ used in questions through a period of being used in self-directed questions or indicating a 
pause within an utterance. It is not clear when it started to be used as a theme marker, but historical data shows that such use had matured in the novels Honglou meng (A Dream of Red Mansions) and Ernü yingxiong zhuan (Legend of the Young Heroes) of the Qing dynasty (seventeenth to early twentieth centuries), when its original use in statements- $l i-$ and that in questions - $n i$ - had merged and become the present $n e$. That is why the particle in this use maintains the two core properties of marking existence in statements and discourse continuation in questions.

As discussed in Section 3, ne in statements marks the existence of a situation missing in the context. Also, as a theme marker, ne marks the concept or referent designated by the host constituent as existing in the shared CG of the interactants, and the speaker's intention to use it as a departure point for the upcoming message.

Existential status is closely linked to locatability, ${ }^{(1)}$ the necessary condition for topic referents or theme concepts (Wu 1998: 11). It is therefore natural for an existence marker to function as a theme marker. And this property of marking existence also distinguishes ne from other topic markers. Pragmatically, since the need for marking something's existence arises only when it is missing, ne does not mark simply any theme, but only a new theme. As Zhang \& Fang (1996: 47) observe, in discourse the particle ne is in general used to make a theme shift, or to indicate a new perspective, and to initiate a textual theme shift.

On the other hand, discourse continuation is an indispensible element of the particle's topic-marking function, which has a clear "looking back for contrast" and "demand to continue" (Chu 2006) effect. Apart from indicating that the host theme is new, as discussed above, the particle ne also signals the relevance of the new theme to the previous one. Again as Zhang \& Fang (1996: 47) observe, as a theme marker $n e$ is not generally used in an utterance initiating a conversation, but is used in a continuing clause or sentence. In discussing $n e$ as a topic marker, Chu (2006: 14) specifies that this function of $n e$ "is not just to instruct the hearer to look back at any thing at all", but "to find something, implicit or explicit, in the preceding discourse that contrasts with the host utterance (or NP) of the $n e$ ". As for "demand to continue", Li (2001: 55) finds that, by marking what precedes it as a new theme, the particle makes it compulsory for a corresponding new rheme to occur so that the new theme-rheme cohere with the preceding theme-rheme both in structure and content. Li's (2001) investigation of data consisting of 1,500 thousand characters has revealed that the majority of the theme-rheme structures marked by ne occur in a context where there is a parallel theme-rheme structure or a proposition back to which the new

\footnotetext{
(1) This term has been borrowed (Wu 1998: 10) from Hawkins' (1978) location theory to describe a cognitive feature of a referent. It indicates whether the referent may be recognized, or located, in an identifiable set in the knowledge shared by the addresser and addressee about the real world or the discourse world, as assessed by the addresser.
} 
theme-rheme structure is semantically related. All of this shows that discourse continuation is indeed the core property of the particle ne as a theme marker. This function is also typically displayed in what are called truncated questions, to which we turn now.

$\mathrm{Wu}$ (2005b) proposes that the so-called truncated question in the form of "NP $n e$ ?" is a third type of question parallel to general questions and Wh-questions (including V-not-V and alternative questions): the thematic question. In such a question the particle $n e$ is more appropriately a theme marker rather than an utterance-final particle, and the function of the question is to raise a new theme and invite the hearer to come up with a rheme according to the preceding context or the shared CG. The main reasons for this position are summarized as follows.

The general view that "NP $n e$ ?" is an elliptical Wh-question is based on the fallacy that the particle $n e$ is the formal marker of Wh-questions and a question marked by $n e$ has to be a Wh-question. The "NP ne?" form can be examined in a different light if this false presupposition is discarded.

A crucial difference between a thematic question and a Wh-question is their focus. A Wh-question always has a specific focus, which falls on the Wh-word, or the question form-V-not-V, or the alternative structure - and which can never be omitted. It has to be the Wh-word even if an elliptical Wh-question has only one word left. The simplest form of a Wh-question with $n e$ is "Wh-word $n e$ ?" On the contrary, what is important in a "NP $n e$ ?" question is the theme, which can never be omitted. A thematic question does not contain a Wh-word or a question form, i.e. it does not express a specific focus at all since its focus depends on the preceding context and the shared CG.

Furthermore, the view of "NP $n e$ ?" questions as "truncated" or "elliptical Wh-questions" goes against the intuition and practice of language users in everyday situations. These terms presuppose an un-truncated or full Wh-question from which a thematic question is then derived through ellipsis. In conversations, however, the reason for using a thematic question is often that the speaker has only just thought of a new theme, but has not formed a full question yet. He may add a full question after the thematic question to specify his point, e.g.

(8) Xiăo Wáng ne ? Tā láile méiyǒu/ma? (小王呢? 他来了没有/吗?)

little Wang-ne? he come-le not/-ma ?

'What about Little Wang? Has he come or not/yet?'

It would be unreasonable in this case to claim that the first question is an elliptical form of the second question. It should also be noted that since a thematic question is open, with no specified question point, it is up to the hearer to interpret it in context and then determine what the question is that is being implied, which can take the form of a Wh-question or general question as long as ne is not taken as a formal marker of 
Wh-questions.

The thematic question reflects the topic-prominent (Li \& Thompson 1974) nature of the Chinese language and the priority it gives to pragmatics over syntax (Liu 1995). The path of the particle in question as it was grammaticalized as a theme marker has exemplified Chao's (1968) insightful observation of the subject-predicate relation in Chinese as question-answer. The thematic question is a basic type of question in the language, and the question form "NP $n i$ [the origin of $n e$ in questions]?" occurred as early as the late Tang and Five Dynasties (seventh to tenth centuries) (Cao 1995: 151-153). From this use of $n i$, the particle was used in self-directed questions and to indicate a pause within an utterance in the Song dynasty (tenth to thirteenth centuries) (Cao 1995: 155-156, Jiang 1986). And it is from these uses developed in the Song dynasty that the particle finally derived its use as a theme marker. With the maturity of that use in the Qing dynasty, the particle in "NP ne?" can be re-analyzed as a theme marker, and this re-analysis has driven home the nature and function of thematic questions. In this formulation, even if a thematic question implies a more specific question, Q, it should occur after the theme marker, i.e. "NP ne, Q?" rather than before it, as in "NP, Q ne?" And since Q is therefore not within the scope of $n e$, it can thus be either a Wh-question or a general one.

In summary, the particle ne used within an utterance functions as a theme marker. In this use it maintains both properties of marking existence in statements and discourse continuation in questions, but only to a lesser degree, since what it marks is only a thematic constituent that does not have the propositional force of a declarative utterance or the interrogative force of a question. The particle ne in so-called "truncated questions" is more appropriately a theme marker rather than an utterance-final particle indicating interrogation. Interrogation in such questions is indicated by intonation and/or the interactional context, as proposed by Shao (1989) and Shi \& Zhang (1995: 82).

\section{The general discourse function of the particle ne}

Do the two functions of ne, of hearer engagement for CG negotiation in statements and discourse continuation in questions, have something in common? Is there something that underpins the seemingly different functions of $n e$ in statements and in questions? The answer is definitely yes. In fact, the two functions entail each other.

The motivation for CG negotiation, or asserting an existing situation in conversation, is that the speaker finds that something highly relevant is absent in the current interaction. This means the use of ne depends on the preceding context and is mainly reactive. Furthermore, the fact that the existing situation asserted by $n e$ in this context overwhelmingly contradicts the addressee's claim or supports the speaker's shows the contextual effect of relevance of the host statement to its context: "the strengthening, and 
the weakening or deletion of an already existing assumption" (Sperber \& Wilson 1986). By asserting an existing situation and signalling its relevance to the interaction, the particle $n e$ does mark discourse continuation.

On the other hand, the close tie between a question and its discourse context and the speaker's epistemic state marked by discourse-continuation marker ne in questions could be interpreted as the existence of the question in the discourse context. Therefore, by marking discourse continuation, ne in questions also implies the question's existing status in its discourse context and in the speaker's epistemic state, which is associated with the speaker's puzzlement and reduced interrogative force in ne questions that is generally recognized in the literature.

It was this link between asserting the existence of a certain situation in response to its absence in discourse (a kind of discourse continuation) and marking a question as a discourse continuation (asserting the question's existence in its interactional context) that made it possible for the original locality marker $l i$ and discourse-continuation marker $n i$ to have a general common function. Historically, the origin of the particle ne in both statements and questions was represented by the same particle - er (Jiang 1986). Then in late Tang and the Five Dynasties this particle $e r$ began to be replaced by $l i$ in statements and by $n i$ in questions. It was only after the Yuan dynasty (thirteenth and fourteenth centuries) that $l i$ and $n i$ were replaced by the particle $n e$, which has been used in both statements and questions ever since. Between the Tang and Yuan dynasties, the use of $l i$ and $n i$ in statements and questions was somehow mixed, and the distinction was never clear-cut (Jiang 1986, Qi 2002).

The fact that the two particles $l i$ and $n i$ share the same origin-er-and that they were both used in statements and in questions at one stage are a clear demonstration of their closely related discourse functions. As both $l i$ and $n i$ underwent the grammaticalization process whereby the primarily concrete and objective meanings of $l i$ (inside) and $n i$ (the way of.../such) became increasingly abstract, interpersonal and speaker-based (Traugott 1989, 1995), their functions also increasingly blended. As a result, both functioned to draw the hearer's attention to the existence of the situation or question designated by the host utterance for discourse continuation. It was this common function that allowed the two markers to finally merge into the particle $n e$ in early Mandarin $(201-1000 \mathrm{CE})$ and modern Chinese (1001-1900).

To sum up, the particle ne has two core properties:

- Existence marking

- Discourse-continuation marking

Existence marking determines that $n e$ is tagged to factual or background information, as 
discussed in Lü (1982), Shi \& Zhang (1995), King (1986) and Xu (2008), rather than to new happenings, which is typically marked by the particle le that has a function of CG updating (Wu 2001, Berg \& Wu 2006). Existence marking also accounts for the particle's function of attracting the hearer's attention as well as the associated speaker's attitude towards the ne utterance, as discussed in Lü (1982: 264) and Ding (1961: 215), since "drawing the hearer's attention" was initially a pragmatic inference from existence marking, and through repeated use has gradually been fossilized as the particle's meaning.

Discourse-continuation marking signals the relevance of a ne utterance to its interactional context. The particle ne primarily instructs the hearer to look back for relevance, ${ }^{(1)}$ which determines that the use of the particle requires an existing discourse or situation, and a ne utterance is always a response to an interactional situation.

The particle ne has one core function:

- Hearer engagement for CG negotiation

It is the interplay between this core function of $n e$ and its context that determines the functions of the particle in statements, questions and as a theme marker discussed above.

(a) In a declarative context the particle draws the hearer's attention to an existing situation designated by the ne utterance with affirmative force. The associated speaker's attitude is often "I'm telling you", "believe me", etc.

(b) In an interrogative context the particle draws the hearer's attention to the existence of a question in the interactional context designated by the ne utterance, and the associated speaker's attitude is often "I wonder", which softens the interrogative force of the question.

(c) Within an utterance the particle draws the speaker's attention to a new theme that is often in contrast with the previous one, and prepares the hearer for the upcoming rheme.

In all three uses what ne does is engage the hearer for CG negotiation in response to a discrepancy in the perceived CG shared by the speaker and hearer. This discrepancy is often manifested by the hearer's lack of a certain piece of information or holding a view different from that of the speaker (to be responded to by a ne statement), or by the speaker's lack of a certain piece of information or holding a view different from that of the hearer (to be responded to by a ne question), or simply by the hearer's being unaware of the speaker's intention to switch to a new theme in contrast to the previous one (to be

\footnotetext{
(1) This is formulated differently from Chu's (2006:23) "Necessity to look back for contrast" in order to accommodate some relatively neutral utterances in terms of “contrast” such as “喝着呢./(?)”, either as a reply to “他在做什么呢?”, or as an initial move to start a conversation. As for Chu's "Demand for continuation", I think that is a feature of a specific (or type of) utterance rather than a property of the particle. For instance, a question, with or without $n e$, demands a reply anyway.
} 
responded to by a $n e$ theme-marking). In a declarative context the speaker uses $n e$ to urge the hearer to adjust her CG by taking what he says as being true and factual, whereas in an interrogative context the speaker urges the hearer to provide information so that he can adjust his CG accordingly.

Other specific interpretations in the literature of the function of a $n e$ utterance such as reminding, emphasizing, contradicting, counter expectation, puzzlement, etc. are all derivable from this core function in context and specific propositional content of the $n e$ utterance.

\section{Conclusion}

This paper has presented a unified account of the discourse function of the Chinese particle $n e$ on the basis of the author's synchronic and diachronic studies on the function of the particle in statements, questions and as a theme marker under an interactional and cognitive framework for discourse markers. It proposes that the particle ne has a general function of hearer engagement for CG negotiation. The use of ne in discourse serves during the joint construction of CG shared by the speaker and hearer in a conversation as an instruction for the hearer to pay special attention to a discrepancy which the speaker perceives as highly relevant to the current interaction and needs to be negotiated and resolved. And it is the interplay between this core function of ne and the propositional content of the host utterance in a specific context that generates the various interpretations in the literature associated with the use of $n e$. This core function of the particle $n e$ is rooted in two core properties - existence marking and discourse-continuation marking-which pertain to its two origins in Middle Chinese (201-1000) and Early Mandarin (1001-1900): the locality marker $l i$ and the discourse-continuation marker $n i$.

\section{References}

Alleton, Viviane. 1981. Final particles and expression of modality in modern Chinese. Journal of Chinese Linguistics 9: 90-115.

Berg, Marinus van den \& Guo Wu. On Chinese Discourse LE: Discourse construction and pragmatic marking in Chinese. London and New York: Routledge.

Cao Guangshun. 1995. Jindai Hanyu Zhuci (Particles in Early Mandarin). Beijing: Yuwen Publishing House.

Chao Yuanren. 1968. A Grammar of Spoken Chinese. Berkeley and Los Angeles: University of California Press.

Chen Meijin. 1995. Beijinghua yiwen yuqici de fenbu, gongneng ji chengyin (The distribution, function and motivation of interrogative particles in Beijing dialect). Zhongguo Yuwen. 1.

Chu, Chauncey. 1999. Hanyu Renzhi Gongneng Yufa (A Cognitive-Functional Grammar of Mandarin Chinese). Taipei: Wenhe Publishing Company Limited.

- 2002. Relevance theory, discourse markers and the Mandarin utterance-final particle A/Ya. Journal of the Chinese Language Teachers Association 37.1: 1-42.

. 2006. A contrastive approach to discourse particles - A case study of the Mandarin UFP Ne 呢. 


\section{Wu Guo}

Journal of Foreign Languages. 3: 7-29.

Clark, H.H. 1996. Using Language. Cambridge: Cambridge University Press.

Ding Shengshu. 1961. Xiandai Hanyu Yufa Jianghua (Lectures on Modern Chinese Grammar). Beijing: Yuwen Publishing House.

Grice, H. Paul. 1971. Meaning. In: Steinberg, D. D. \& L. A. Jakobovits (eds.), Semantics: 53-59. Cambridge: Cambridge University Press.

- 1975. Logic and conversation. In: Peter Cole and Jerry L. Morgan (eds.), Syntax and Semantics 3: 41-58. New York: Academic Press.

Halliday, M.A.K. 1985. An introduction to functional grammar. London ; Baltimore, Md., USA : Edward Arnold.

Harder, Peter. 1991. Linguistic meaning: cognition, interaction and the real world. Nordic Journal of Linguistics 14: 119-140.

- 1996. Functional Semantics. A Theory of Meaning, Structure and Tense in English. Berlin: Mouton de Gruyter.

Heritage, John. 1984. Garfinkel and Ethnomethodology. Cambridge: Polity Press.

$\mathrm{Hu}$ Mingyang. 1981. Beijinghua de yuqici he tanci (Particles in Beijing dialect). Zhongguo Yuwen. 5:347-350, 6: 416-423.

Jiang Lansheng. 1986. Yiwen yuqici "ne" de laiyuan (The origin of the interrogative particle ne). Yuwen Yanjiu. 2.

Jiang Shaoyu. 2005. Jindai Hanyu Yanjiu Gaiyao (An Overview of Early Mandarin Research). Beijing: Peking University Press.

Johnson-Laird, P.N. 1977. Procedural semantics. Cognition 5: 189-214.

1983. Mental Models. Cambridge: Cambridge University Press.

1989. Mental models. In: Michael I. Posner (ed.), Foundations of Cognitive Science: 469-499. Cambridge, MA: MIT Press.

Kang Liangfang. 1998. Cong xiandai hanyu yiwenju de goucheng qingkuang kan yiwenju jumo yuqici "ne" (The structure of questions in modern Chinese and the utterance-final particle ne). Journal of Sichuan University (Social Science edition). 25, 4: 93-98.

King, Brian. 1986. ne-A discourse approach. Journal of the Chinese Language Teachers Association 21.1: 21-46.

Li Daqin. 2001. "WP ne?" wenju yiwen gongneng de chengyin shixi (An analysis of the origin of the "WP ne?" questions). Language Teaching and Linguistic Studies. 6: 52-61.

Li, Ing Cherry. 1999. Utterance-Final Particles in Taiwanese: A Discourse-Pragmatic Analysis. Taipei: Crane Publishing.

Li, Charles N. \& Sandra A. Thompson. 1981. Mandarin Chinese: A Functional Reference Grammar. Berkeley and Los Angeles: University of California Press.

- 1974. Chinese as a topic-prominent language. Unpublished paper presented at the $7^{\text {th }}$ International Conference on Sino-Tibetan Languages and Linguistics, Atlanta, Georgia.

Liu Danqing. 1995. Yuyi youxian haishi yuyong youxian - Hanyu yufa tixi jianshe duanxiang (Priority on semantics or pragmatics? - thoughts on the construction of a Chinese grammatical system). Yuwen Yanjiu. 2: 10-15.

Liu Yuehua et al. 1983. Shiyong Xiandai Hanyu Yufa (A Practical Grammar of Modern Chinese). Beijing: Foreign Language Teaching and Research Press.

Lü Shuxiang. (ed.) 1980. Xiandai Hanyu Babai ci (Eight Hundred Words in Modern Chinese). Beijing: Commercial Press.

Mosegaard Hansen, Maj-Britt. 1998. The Function of Discourse Particles. Amsterdam: John Benjamins Publishing Company.

Ohta, Tatsuo. 1987. Zhongguo Lishi Yufa (A Historical Study of the Chinese Language). (Translated by Jiang Shaoyu and Xu Changhua). Beijing: Peking University Press.

Qi Huyang. 2002. Yuqici yu Yuqi Xitong (Modal Particles and Modality). Hefei: Anhui Education Press.

Schiffrin, Deborah. 1987. Discourse Markers. Cambridge: Cambridge University Press. - 1994. Approaches to Discourse. Cambridge: Blackwell. 


\section{A Unified Account of the Discourse Function of the Chinese Particle "ne"}

Shao Jingmin. 1989. Yuqici "ne" zai yiwenju zhong de zuoyong (The function of the particle ne in questions). Zhongguo Yuwen. 3: 170-175.

Shi Yuzhi and Zhang Ping. 1995. 'ne' de yufa yiyi ji yu yiwen daici gongxian de tiaojian (The grammatical meaning of $n e$ and conditions for $n e$ 's co-occurrence with wh-words). JCLTA. 30. 2: 71-83.

Sperber, Dan \& Deirdre Wilson. 1986. Relevance: Communication and Cognition. Oxford: Blackwell.

Sun Xixin. 1997. Hanyu Lishi Yufa Conggao (Drafts of Historical Chinese Grammar). Beijing: Chinese Dictionary Press.

Traugott, E. Closs. 1989. On the Rise of Epistemic Meaning in English. Language, 65: 31-55.

- 1995. "Subjectification in grammaticalisation". In Stein, Dieter and Susan Wright (eds.) Subjectivity and subjecivisation: linguistic perspectives. Cambridge: Cambridge University Press.

Tsao Fengfu. 2000. Huayu xuzi de yanjiu yu jiaoxue - Yi 'ne' zi wei li (Chinese particles teaching and research - An example of 'ne'). Collected Papers of the $6^{\text {th }}$ World Symposium on Chinese Language Teaching and Research. Taipei: The World Association of Chinese Language Teaching.

Wu Guo (武果). 1998. Information Structure in Chinese. Beijing: Peking University Press.

- 2001. A new approach to an old problem: on Chinese discourse $L E$. In Husken, Frans \& Dick van der Meij (eds.), Reading Asia: new research in Asia studies. 261-278. Richmond: Curzon Press.

- 2005a. The Discourse Function of the Chinese Particle NE in Statements". Journal of Chinese Language Teachers Association. Feb. 47-81.

. 2005b. Yiwenju zhong de " $n$ " (The particle ne in questions). Chinese Language Research and Application. 3: 64-82.

2006. "Zhuweiwen" - tan "feiyiwen xingshi + ne?" yiwenju (The "thematic question" - On "non-interrogative constituent + particle ne" questions). Linguistics Forum. 32: 64-82.

Xu Jingning. 2008. Xiandai Hanyu huayu qingtai yanjiu (A Study of Discourse Modality in Modern Chinese). Beijing: Kunlun Publishing House.

Yang Shuda. 1928. Ci Quan (A Glossary). Beijing: Commercial Press.

1929. Gaodeng Guowenfa (An Advanced Chinese Grammar). Beijing: Commercial Press.

Ye Rong. 1994. Guanyu feishifei wenju li de "ne" (On the particle ne in non-general questions). Zhongguo Yuwen. 6: 448-451.

Yu Shiwen et al. 1998. Xiandai Hanyu yufa xinxi cidian xiangjie (A Dictionary of Modern Chinese Grammar). Beijing: Qinghua University Press.

Zhang Bojiang and Fang Mei. 1996. Hanyu Gongneng Yufa Yanjiu (A Study of Chinese Functional Grammar). Nanchang: Jiangxi Education Press.

Zhu Dexi. 1982. Yufa Jiangyi (Lectures on Chinese Grammar). Beijing: Commercial Press. 